PROCEEDINGS OF THE

AMERICAN MATHEMATICAL SOCIETY

Volume 137, Number 5, May 2009, Pages 1771-1776

S 0002-9939(08)09709-8

Article electronically published on December 10, 2008

\title{
ON PERIOD MINIMAL PSEUDO-ANOSOV BRAIDS
}

\author{
ANDRÉ DE CARVALHO, TOBY HALL, AND RUPERT VENZKE
}

(Communicated by Bryna Kra)

\begin{abstract}
A family of period minimal pseudo-Anosov braids, one for each pair of Farey neighbors in $(0,1 / 2]$, is described.
\end{abstract}

\section{INTRODUCTION}

When studying the forcing order on the set of braid types, it is natural to consider those pseudo-Anosov braids which are in some sense minimal: that is, they force as little dynamics as possible.

Perhaps the most natural notion of minimality is provided by topological entropy: a pseudo-Anosov $n$-braid $\beta$ is entropy minimal if $h(\beta) \leq h(\gamma)$ for all pseudo-Anosov $n$-braids $\gamma$, where $h(\beta)$ denotes the topological entropy of the pseudo-Anosov representative of $\beta$. Ham and Song HS07] describe entropy minimal $n$-braids for $n \leq 5$ (the result is trivial for $n \leq 3$, and was originally stated for $n=4$ by Song, Ko, and Los SKL02]). However their proof, which involves a computer-assisted search of train track automata, does not offer any immediate prospect of extension to much higher values of $n$ : recent work by Cho and Ham $\mathrm{CH} 08$ on entropy minimal pseudo-Anosovs on the genus two surface may extend to provide an entropy minimal 6-braid.

Another notion of minimality arises from considering the periods of braids forced by periodic pseudo-Anosov braids. A periodic pseudo-Anosov $n$-braid (i.e., one whose underlying permutation is an $n$-cycle) is period minimal if it doesn't force any other periodic pseudo-Anosov $k$-braids with $k \leq n$. This is related to the notion of primary braids studied by Franks and Misiurewicz [FM93]: a periodic $n$-braid is primary if it forces no other periodic $n$-braids. However, no examples of primary pseudo-Anosov braids are known. Indeed, recent work of le Calvez establishes that there are no primary pseudo-Anosov braids of prime period. He shows that every periodic orbit of an orientation-preserving disk homeomorphism has non-zero rotation number about some fixed point; it follows from a result of Boyland Boy92 that if the period of the orbit is prime, then the homeomorphism must have a periodic orbit of finite order type of the same period.

The purpose of this paper is to observe that results of dCH04 provide a family of period minimal pseudo-Anosov braids, one for each pair of Farey neighbors in $(0,1 / 2]$.

Received by the editors July 15, 2008.

2000 Mathematics Subject Classification. Primary 37E30.

The first author is grateful for support from CNPq grant No. 309679/2007-9 and FAPESP grant No. 2006/03829-2.

(C) 2008 American Mathematical Society 1771

Reverts to public domain 28 years from publication 
The necessary background to state and prove this result is summarised in Section 2, and the short proof itself is given in Section 3. The exposition allows the reader to construct the minimal braids concerned without having to understand the details of the underlying theory; see Example 3.3 .

\section{HORSESHOE BRAIDS}

2.1. Braid types and forcing. In this section, Boyland's definitions of braid type and braid type forcing Boy 84 are recalled.

Let $f: D^{2} \rightarrow D^{2}$ be an orientation-preserving homeomorphism, and $P$ be a (least) period $n$ orbit of $f$. The braid type bt $(P, f)$ of $P$ is the isotopy class of $f$ relative to $P$ up to an orientation-preserving topological change of coordinates. That is, bt $(P, f)$ is a conjugacy class in $B_{n} / Z\left(B_{n}\right)$, where $B_{n}$ is Artin's $n$-braid group, whose center $Z\left(B_{n}\right)$ is the infinite cyclic subgroup generated by a full twist.

To find a representative braid in this conjugacy class, pick an orientationpreserving homeomorphism $h:\left(D^{2}, P\right) \rightarrow\left(D^{2}, A_{n}\right)$, where $A_{n}$ is a standard $n$ element subset of $D^{2}$ whose points all lie on the horizontal diameter of the disk. Let $g=h \circ f \circ h^{-1}:\left(D^{2}, A_{n}\right) \rightarrow\left(D^{2}, A_{n}\right)$, and pick an isotopy $\left\{g_{t}\right\}:$ id $\simeq g$ (not relative to $A_{n}$ ). Then the subset

$$
\bigcup_{t \in[0,1]} g_{t}\left(A_{n}\right) \times\{t\}
$$

of $D^{2} \times[0,1]$ is a geometric braid $\beta \in B_{n}$, and $\beta Z\left(B_{n}\right) \in \operatorname{bt}(P, f)$.

Because $B_{n} / Z\left(B_{n}\right)$ is isomorphic to the mapping class group of the $n$-punctured disk, braid types can be classified as being of pseudo-Anosov, reducible, or finite order braid type according to Thurston's classification [Thu88, [FLP91, CB88.

Let BT denote the set of all braid types. The forcing relation is a partial order $\leq$ on BT, defined as follows: if $\beta, \gamma \in \mathrm{BT}$, then $\beta \leq \gamma(\gamma$ forces $\beta)$ if and only if every orientation-preserving homeomorphism $f: D^{2} \rightarrow D^{2}$ which has a periodic orbit of braid type $\gamma$ also has one of braid type $\beta$. Thus the forcing relation provides information on the order in which periodic orbits can be created in parameterised families of homeomorphisms.

2.2. Periodic orbits of Smale's horseshoe map. The braid types considered in this paper come from periodic orbits of Smale's horseshoe map $F: D^{2} \rightarrow D^{2}$ Sma67, whose action (on a stadium-shaped subset of the disk) is depicted in Figure 1.

Any period $N$ orbit $P$ of $F$ (except for the fixed point in the left-hand semi-circle of the stadium) is contained entirely in the square $S$ and can be described by its code $c=c_{P} \in\{0,1\}^{N}$ : let $x$ be the rightmost point of $P$, and for $0 \leq i<N$ let $c_{i}=0$ if $F^{i}(x)$ lies in the left-hand side of $S$, and $c_{i}=1$ if $F^{i}(x)$ lies in the right-hand side of $S$.

Let HS denote the set of braid types of periodic orbits of $F$. Note that if $\gamma \in \mathrm{HS}$ and $\beta \leq \gamma$, then $\beta \in \mathrm{HS}$, since by definition $F$ has a periodic orbit of braid type $\beta$.

Representative braids of elements of HS are permutation braids of permutations $\pi \in S_{N}$ which are unimodal (i.e., $\pi(i)<\pi(j)$ if $1 \leq i<j \leq \pi^{-1}(N)$ or if $\left.\pi^{-1}(N) \leq j<i \leq N\right)$. The relevant permutation can be determined from the code 


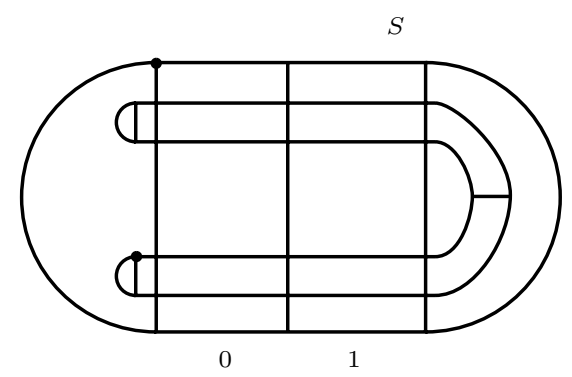

Figure 1. Smale's horseshoe map

of a periodic orbit using the unimodal order, which is a total order $\preceq$ on $\{0,1\}^{\mathbb{N}}$ defined by $s \preceq t$ if and only if either $s=t$, or $s_{0} s_{1} \ldots s_{k}$ contains an even number of $1 \mathrm{~s}$, where $k$ is least such that $s_{k} \neq t_{k}$.

If $P$ is a period $N$ horseshoe orbit, order the $N$ elements $\sigma^{i}\left(c_{P}^{\infty}\right)(0 \leq i<N)$ of $\{0,1\}^{\mathbb{N}}$ using the unimodal order (here $c_{P}^{\infty}$ is the element of $\{0,1\}^{\mathbb{N}}$ obtained by repeating the word $c_{P}$ infinitely often, and $\sigma:\{0,1\}^{\mathbb{N}} \rightarrow\{0,1\}^{\mathbb{N}}$ is the shift map which removes the first symbol of its input). Let $\sigma^{i}\left(c_{P}^{\infty}\right)$ be $r_{i}^{\text {th }}$ in the order. Then a representative braid $\beta_{P}$ of the braid type of $P$ is the permutation braid of the permutation $\pi_{P}$ given by $\pi_{P}\left(r_{i}\right)=r_{i+1} \bmod N$.

Example 2.1. Let $c_{P}=10010$. Then the elements of $\sigma^{i}\left(c_{P}^{\infty}\right)$ and their relative orderings are as given in the table on the left of Figure 2 hence $\pi_{P}=(12435)$, and $\beta_{P}$ is the permutation braid of $\pi_{P}$, i.e. $\beta_{P}=\sigma_{3} \sigma_{2} \sigma_{4} \sigma_{3} \sigma_{2} \sigma_{1}$, as depicted on the right of Figure 2,

\begin{tabular}{|c|c|c|}
\hline$i$ & $\sigma^{i}\left(c_{P}^{\infty}\right)$ & $r_{i}$ \\
\hline 0 & $1001010010 \ldots$ & 5 \\
1 & $0010100101 \ldots$ & 1 \\
2 & $0101001010 \ldots$ & 2 \\
3 & $1010010100 \ldots$ & 4 \\
4 & $0100101001 \ldots$ & 3 \\
\hline
\end{tabular}

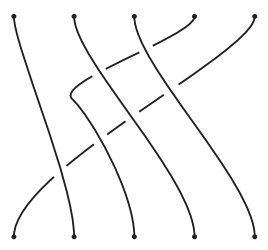

FiguRE 2. Calculating a horseshoe braid

Every non-fixed periodic orbit $P$ of $F$ has a rotation number $\rho(P) \in(0,1 / 2] \cap \mathbb{Q}$, which is its rotation number in the annulus obtained by puncturing the disk at the fixed point of $F$ with code 1 . The rotation number is a braid type invariant, and hence it is possible to define the rotation interval $\rho i(\gamma)$ of a period $N \geq 2$ braid type $\gamma \in$ HS by

$$
\rho i(\gamma)=\{\rho(\beta): \beta \leq \gamma \text { and } \beta \text { has period } \geq 2\} .
$$

The rotation interval is a closed interval in $\mathbb{Q}$ with rational endpoints Han90. Its endpoints are distinct if $\gamma$ is of pseudo-Anosov type. 
$(0,0)$

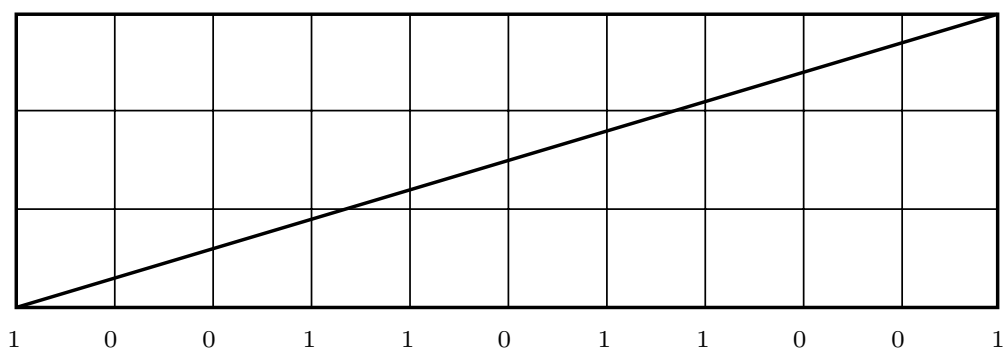

$(10,3)$

Figure 3 . The words $c_{3 / 10}$ and $\tilde{c}_{3 / 10}$

2.3. Star periodic orbits of the horseshoe. The minimal braids described in this paper are associated to the so-called star braid types of the horseshoe, which are considered in [dCH04]. There is one such braid type $\beta_{u / v}^{m / n}$ for each pair of rationals $u / v<m / n \in(0,1 / 2]$.

In order to describe these braid types, it is necessary first to define, for each rational $m / n \in(0,1 / 2]$, words $c_{m / n} \in\{0,1\}^{n+1}$ and $\tilde{c}_{m / n} \in\{0,1\}^{n-1}$. Let $L_{m / n}$ be the straight line in $\mathbb{R}^{2}$ from $(0,0)$ to $(n, m)$. For $0 \leq i \leq n$, let $s_{i}=1$ if $L_{m / n}$ crosses some line $y=$ integer for $x \in(i-1, i+1)$, and $s_{i}=0$ otherwise. Then $c_{m / n}=s_{0} s_{1} \ldots s_{n}$, and $\tilde{c}_{m / n}=s_{1} \ldots s_{n-1}$.

Equivalently, define integers $\kappa_{i}$ for $1 \leq i \leq m$ by $\kappa_{1}=\lfloor n / m\rfloor-1$ and $\kappa_{i}=$ $\lfloor i n / m\rfloor-\lfloor(i-1) n / m\rfloor-2$ for $2 \leq i \leq m$, where $\lfloor x\rfloor$ is the integer part of $x$. Then $\tilde{c}_{m / n}=0^{\kappa_{1}} 110^{\kappa_{2}} 11 \ldots 110^{\kappa_{m}}$, and $c_{m / n}=1 \tilde{c}_{m / n} 1$.

Example 2.2. From Figure 3, $c_{3 / 10}=10011011001$ and $\tilde{c}_{3 / 10}=001101100$. The integers $\kappa_{i}$ are given by $\kappa_{1}=\lfloor 10 / 3\rfloor-1=2, \kappa_{2}=\lfloor 20 / 3\rfloor-\lfloor 10 / 3\rfloor-2=1$, and $\kappa_{3}=\lfloor 30 / 3\rfloor-\lfloor 20 / 3\rfloor-2=2$.

Given $0<u / v<m / n \leq 1 / 2$, define $c_{u / v}^{m / n}$ to be the length $v+n$ word $c_{u / v} \tilde{c}_{m / n}$. The following lemma summarises the relevant results of dCH04:

Lemma 2.3. Let $u / v, m / n \in \mathbb{Q} \cap(0,1 / 2]$ with $u / v<m / n$. Then $c_{u / v}^{m / n}$ is the code of a period $v+n$ horseshoe orbit of pseudo-Anosov braid type.

Denote the braid type of this periodic orbit by $\beta_{u / v}^{m / n}$.

Remark 2.4. Changing the initial symbol of $\tilde{c}_{m / n}$ from 0 to 1 yields (except in the case $m / n=1 / 2$ ) a distinct braid which is, however, conjugate to that given by the code $c_{u / v} \tilde{c}_{m / n}$, and hence defines the same braid type $\beta_{u / v}^{m / n}$ dCH03.

\section{A FAMILY OF PERIOD MINIMAL BRAIDS}

The main result of this paper is an easy consequence of the following, which is Lemma 17 of $\mathrm{dCH} 04$.

Lemma 3.1. Let $\beta$ be a period $N$ horseshoe braid type with non-trivial rotation interval $\rho i(\beta)=[u / v, m / n]$. Then $N \geq v+n$. Moreover, $N=v+n$ if and only if $\beta=\beta_{u / v}^{m / n}$. 
Theorem 3.2. Let $u / v<m / n$ be rationals in $(0,1 / 2]$ which are Farey neighbors. Then the braid type $\beta_{u / v}^{m / n}$ is pseudo-Anosov and is period minimal.

Proof. $\beta=\beta_{u / v}^{m / n}$ is a pseudo-Anosov braid type of period $N=v+n$ by Lemma 2.3 . If $\beta \geq \gamma$ and $\gamma$ is pseudo-Anosov, then $\gamma$ is a horseshoe braid type with nontrivial rotation interval $\rho i(\gamma) \subseteq \rho i(\beta)=[u / v, m / n]$. Since $u / v$ and $m / n$ are Farey neighbors, it follows that either $\rho i(\gamma)=[u / v, m / n]$ (and hence $\gamma=\beta$ by Lemma3.1) or $\rho i(\gamma)=\left[u^{\prime} / v^{\prime}, m^{\prime} / n^{\prime}\right]$, where $v^{\prime}+n^{\prime}>v+n=N$. In the latter case, $\gamma$ has period greater than $N$ by Lemma 3.1 .

Example 3.3. Let $(u / v, m / n)=(1 / 4,2 / 7)$, so $c_{u / v}=10001$ and $\tilde{c}_{m / n}=001100$. Hence 10001001100 is the code of a period 11 horseshoe orbit of period minimal braid type. The corresponding horseshoe braid is the permutation braid of the permutation (1361025894711).

Similarly, let $(u / v, m / n)=(1 / 3,3 / 8)$, so $c_{u / v}=1001$ and $\tilde{c}_{m / n}=0110110$. Hence 10010110110 is the code of another period 11 horseshoe orbit of period minimal braid type, distinct from the previous one since it has a different rotation interval. The corresponding horseshoe braid is the permutation braid of the permutation (1410269378511).

\section{REFERENCES}

[Boy84] P. Boyland, Braid types and a topological method of proving positive entropy, preprint, Boston University (the results of this preprint are included in the published paper Boy94), 1984.

[Boy92] , Rotation sets and monotone periodic orbits for annulus homeomorphisms, Comment. Math. Helv. 67 (1992), no. 2, 203-213. MR.1161281 (93k:58180)

[Boy94] , Topological methods in surface dynamics, Topology Appl. 58 (1994), no. 3, 223-298. MR:1288300 (95h:57016)

[CB88] A. Casson and S. Bleiler, Automorphisms of surfaces after Nielsen and Thurston, London Mathematical Society Student Texts, vol. 9, Cambridge University Press, Cambridge, 1988. MR 964685 (89k:57025)

[CH08] J. Cho and J-Y. Ham, The minimal dilatation of a genus two surface, Experiment. Math. 17 (2008), no. 3, 257-267.

[dCH03] A. de Carvalho and T. Hall, Conjugacies between horseshoe braids, Nonlinearity 16 (2003), no. 4, 1329-1338. MR1986298 (2005b:37071)

[dCH04] _ Braid forcing and star-shaped train tracks, Topology 43 (2004), no. 2, 247-287. MR2052964 (2005c:37075)

[FLP91] Travaux de Thurston sur les surfaces, Société Mathématique de France, Paris, 1991, Séminaire Orsay, reprint of Travaux de Thurston sur les surfaces, Soc. Math. France, Paris, 1979, MR0568308 (82m:57003) Astérisque No. 66-67 (1991). MR.1134426 (92g:57001)

[FM93] J. Franks and M. Misiurewicz, Cycles for disk homeomorphisms and thick trees, Nielsen theory and dynamical systems (South Hadley, MA, 1992), Amer. Math. Soc., Providence, RI, 1993, pp. 69-139. MR95e:58133

[Han90] M. Handel, The rotation set of a homeomorphism of the annulus is closed, Comm. Math. Phys. 127 (1990), no. 2, 339-349. MR1037109 (91a:58102)

[HS07] J-Y. Ham and W. Song, The minimum dilatation of pseudo-Anosov 5-braids, Experiment. Math. 16 (2007), no. 2, 167-179. MR2339273

[SKL02] W. Song, K. Ko, and J. Los, Entropies of braids, J. Knot Theory Ramifications 11 (2002), no. 4, 647-666, Knots 2000 Korea, Vol. 2 (Yongpyong). MR1915500 (2003e:57013) 
[Sma67] S. Smale, Differentiable dynamical systems, Bull. Amer. Math. Soc. 73 (1967), 747-817. MR $37: 3598$

[Thu88] W. Thurston, On the geometry and dynamics of diffeomorphisms of surfaces, Bull. Amer. Math. Soc. (N.S.) 19 (1988), no. 2, 417-431. MR89k:57023

Departamento de Matemática Aplicada, ime-Usp, Rua do Matão 1010, Cidade UniVersitária, 05508-090 São Paulo, SP, Brazil

Department of Mathematical Sciences, University of Liverpool, Liverpool L69 7ZL, United Kingdom

Department of Mathematics, California Institute of Technology, Pasadena, CaliFORNIA 91125

Current address: Department of Mathematics, Northwestern University, 2033 Sheridan Road, Evanston, Illinois 60208 